\title{
Bridging molecular and cellular biology with optics (Presentation Video)
}

\section{Gabriel Popescu}

Gabriel Popescu, "Bridging molecular and cellular biology with optics (Presentation Video)," Proc. SPIE 9339, Reporters, Markers, Dyes, Nanoparticles, and Molecular Probes for Biomedical Applications VII, 933902 (27 April 2015); doi: 10.1117/12.2179067

SPIE. Event: SPIE BiOS, 2015, San Francisco, California, United States 


\title{
Bridging molecular and cellular biology with optics (Presentation Video)
}

\author{
Gabriel Popescu, University of Illinois at Urbana-Champaign (USA)
}

\begin{abstract}
Understanding the structure and function of living cells from the nano to the micron scale remains a grand challenge. While X-ray diffraction and electron microscopy reveal nanoscale information from cellular structures, they operate with lifeless specimens. By contrast, optical techniques are well suitable for studying live cells. However, the resolution of the far-field "linear" microscopy is approximately $300 \mathrm{~nm}$, a manifestation of Heisenberg's uncertainty relation. Thus, pushing the biophotonics investigation toward the molecular scale is faced with significant challenges but also with unique opportunities. We will describe various principles dedicated to this goal and present some recent advances in phase sensitive measurements.
\end{abstract}

View presentation video on SPIE's Digital Library: http://dx.doi.org/10.1117/12.2179067.4093525328001 